\title{
Évaluation clinique de la sécrétion rénale de potassium : une nouvelle méthode non invasive
}

\section{Jean Ethier \\ Peter O. Magner \\ Kamel S. Kamel \\ Michael L. West \\ Mitchell L. Halperin}

\section{ADRESSES \\ M.L. Halperin : Professeur de médecine, uni- versité de Toronto. Lab. \#l, Research Wing, St. Michael's Hospital, 38 Shuter Street, Toronto, Ontario, Canada M5B l A6. \\ J. Ethier: Stagiaire de recherche, université de Toronto. P.O. Magner : Stagiaire de recherche, université de Toronto. K.S. Kamel : Professeur de médecine, université de Saskatchewan. M.L. West. Service de néphrologie, St. Michael's Hospital, Toronto, Ontario, Canada.}

\section{TIRÉS A PART}

M.L. Halperin

J. Ethier est boursier du Fonds de la recherche en santé du Québec.

P.O. Magner et K.S. Kamel sont boursiers de la Fondation canadienne des maladies du rein.

$\mathrm{m} / \mathrm{s} n^{\circ} 10$ vol. 4 , décembre 88

La sécrétion de potassium (K) se fait au niveau du «néphron cortical distal ». Elle dépend de l'action de l'aldostérone, du débit urinaire et exige un apport suffisant de sodium à cette région du néphron. Le gradient de concentration trans-tubulaire de potassium (GTTK) est un paramètre facile à calculer à partir des osmolalités du plasma et de l'urine et des concentrations du potassium urinaire et plasmatique. Lorsque le GTTK est supérieur à 7 , on peut être assuré que la sécrétion d'aldostérone et la sensibilité rénale à cette hormone permettent une sécrétion active de $K$. En revanche, une valeur inférieure à 4 de ce paramètre indique que l'activité sécrétoire est faible.

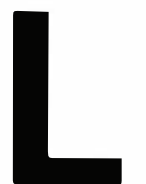

a concentration du potassium $(\mathrm{K})$ dans le liquide extracellulaire est un déterminant majeur du potentiel de repos des membranes cellulaires et, donc, de l'excitabilité neuromusculaire. L'hyperkaliémie ainsi que l'hypokaliémie, deux désordres électrolytiques fréquents, peuvent donc entraîner des arythmies potentiellement mortelles.

Une concentration anormale de $\mathrm{K}$ dans le liquide extracellulaire peut être secondaire soit à un déplacement de $\mathrm{K}$ entre les liquides intra- et extracellulaires (généralement de courte durée), soit à une modification dans le contenu corporel total en $\mathrm{K}$. Ce dernier problème est généralement dû à des anomalies de l'excrétion rénale du $\mathrm{K}$; cependant, des pertes extrarénales anormales ou un apport exagéré en $\mathrm{K}$ jouent parfois un rôle étiologique important.

\section{Physiologie de l'excrétion du potassium}

L'excrétion de $\mathrm{K}$ est réglée par deux groupes de facteurs principaux: ceux qui influencent la concentra- tion de $\mathrm{K}$ dans l'urine (par exemple l'aldostérone) et ceux qui influencent le volume urinaire (par exemple la charge hydrique et sodée) (équation l) :

$\mathrm{K}$ excrété $=[\mathrm{K}]$ urinaire $\times$ volume urinaire (l)

En plus de ces facteurs principaux de régulation, deux facteurs permissifs jouent un rôle dans certaines circonstances : l'apport de sodium ( $\mathrm{Na}$ ) au niveau du tubule distal et l'hormone antidiurétique $(\mathrm{ADH})[1]$.

Mécanisme de sécrétion du potassium (figure 1). La sécrétion de $\mathrm{K}$ se fait au niveau du tubule contourné distal, dans sa portion terminale, et du tubule collecteur cortical (segment dénommé « néphron distal cortical »). Le processus est déclenché par la réabsorption du sodium luminal par les cellules tubulaires principales. Ce mouvement, entraîné par le gradient électrochimique de $\mathrm{Na}$ de part et d'autre de la membrane luminale, se fait via un canal sodique sélectif. Dans la cellule, et sous l'effet de la $\mathrm{Na}^{+}-\mathrm{K}^{+}$ATPase basolatérale, trois $\mathrm{Na}^{+}$sont transportés vers le liquide extracellulaire en échange de deux $\mathrm{K}^{+}$. Cette étape - outre qu'elle préserve le gradient de concentration 


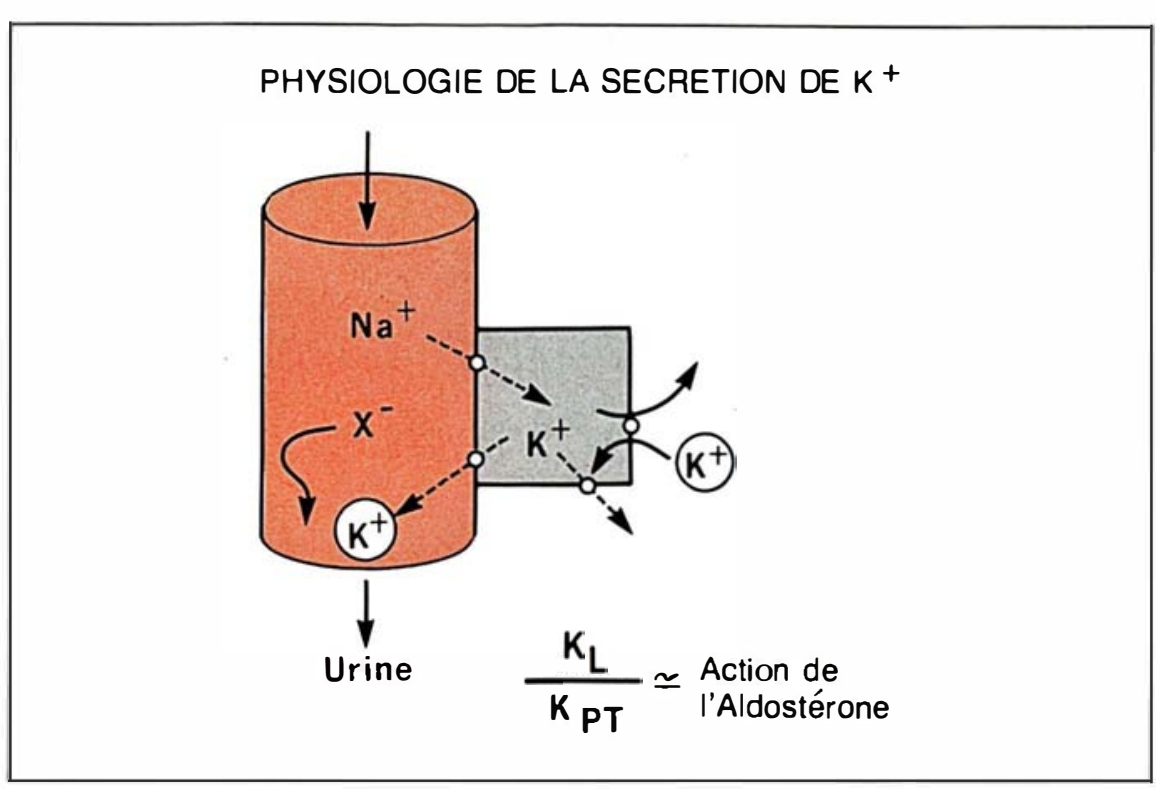

Figure 1. Physiologie de la sécrétion de $K$ induite par l'aldostérone. La structure cylindrique (en rouge) représente la lumière du tubule contourné distal dans sa portion terminale ou celle du tubule collecteur cortical; le carré (en gris) représente une cellule principale. Le premier effet de l'aldostérone est d'ouvrir le canal luminal au $\mathrm{Na}$; le Na pénètre alors dans la cellule selon son gradient, laissant derrière l'anion X. Ceci augmente l'électronégativité de la lumière. Le Na intracellulaire est alors pompé hors de la cellule par la $\mathrm{Na}^{+}-\mathrm{K}^{+}$ATPase de la membrane basolatérale, ce qui augmente la concentration intracellulaire en $K$. Certains de ces ions $K$ passent alors dans la lumière à cause du gradient électrique. Le rapport de concentration de $K$ dans la lumière $(K)$ et dans l'espace péritubulaire $\left(K_{P T}\right)$ devrait être un indice du processus sécrétoire de $K$ (habituellement stimulé par l'aldostérone).

luminale de sodium - augmente le contenu intracellulaire en $\mathrm{K}$. Certains de ces ions $\mathrm{K}$ diffusent dans la lumière tubulaire par des canaux spécifiques. L'importance de cette diffusion dépend de la différence de potentiel transépithéliale engendrée par la réabsorption de sodium sans l'anion qui l'accompagne. Il est à noter que, dans ce segment du néphron, le chlore n'est que partiellement réabsorbable. L'aldostérone intervient dans ce système à quatre niveaux: (1) son action principale est d'ouvrir les canaux sodiques luminaux (ceux-ci sont sensibles à l'effet de l'amiloride); (2) elle augmente l'activité de la $\mathrm{Na}^{+}-\mathrm{K}^{+}$ATPase basolatérale; (3) elle augmente la conductance de la membrane luminale au K; (4) elle diminue la conductance luminale a chlore [2]. Lorsque l'effet de l'aldostérone est

transépithéliale est de $-50 \mathrm{mV}$ et correspond à un rapport de concentration, $\mathrm{K}$ tubulaire/K péritubulaire, environ égal à 10.

Contrôle de l'excrétion rénale de potassium. Examinons comment les deux facteurs principaux et les deux facteurs permissifs influencent l'excrétion de K. Notons d'abord que le mécanisme de sécrétion du $\mathrm{K}$ détermine la concentration luminale en $\mathrm{K}$, premier facteur principal (équation l). Cette concentration, sous l'effet de l'aldostérone, représente environ dix fois celle du plasma et, normalement, n'est pas influencée par le volume urinaire. Le second facteur principal est le volume de liquide s'écoulant dans le tubule collecteur; s'il double, alors que la diffusion du $\mathrm{K}$ assure une concentration tubulaire en $K$ constante, l'excrétion de $\mathrm{K}$ doublera aussi
(Tableau $I b$ ). Donc, ces deux facteurs «primaires » déterminent la kaliurèse. Le rôle des deux facteurs permissifs peut être présenté de la façon suivante: la présence de sodium luminal est nécessaire pour déclencher (mais non pour contrôler) le processus de sécrétion du K (le contrôle du transport du sodium se fait par l'ouverture ou non de canaux sodiques luminaux, en grande partie sous le contrôle de l'aldostérone). La concentration luminale en sodium, requise pour atteindre $50 \%$ du taux maximal de sécrétion de $\mathrm{K}$ chez le rat, est de $13 \mathrm{mmol} / 1[3]$. Le deuxième facteur permissif, l'hormone antidiurétique $(\mathrm{ADH})$, contribue à garder les canaux potassiques ouverts dans la membrane [4] (ces canaux sont habituellement ouverts et ne règlent pas mais permettent la sécrétion de $\mathrm{K}$ ). L'importance du rôle de l'ADH est mise en lumière dans les cas de diabète insipide central (c'est-à-dire en l'absence d'ADH). Dans cette circonstance, le volume urinaire est très élevé ; si l'urine avait une concentration élevée en $\mathrm{K}$, la quantité de $\mathrm{K}$ excrétée pourrait entraîner rapidement une hypokaliémie sévère. L'absence d'ADH diminue la conductance membranaire au $\mathrm{K}$ et protège donc contre une déplétion potassique grave.

\section{Test d'évaluation de l'excrétion rénale de potassium}

Le clinicien dispose de plusieurs épreuves de laboratoire lui permettant d'évaluer les désordres de la kaliémie. Nous les passerons brièvement en revue.

K urinaire de 24 heures. L'examen des urines de 24 heures permet de déterminer si la réponse rénale à un désordre de la kaliémie est adéquate. Une excrétion urinaire de $\mathrm{K}$ normale devrait être inférieure à $30 \mathrm{mmol}$ par jour en présence d'une hypokaliémie et supérieure à $100 \mathrm{mmol}$ par jour en présence d'une hyperkaliémie. Malheureusement, les résultats de l'examen des urines de 24 heures ne sont pas immédiatement disponibles et, si la réponse rénale est inappropriée au contexte clinique, ils ne permettent pas d'identifier de façon exacte le mécanisme d'excrétion responsable 
Tableau I

FACTEURS POUVANT AUGMENTER L'EXCRÉTION RÉNALE DE POTASSIUM

\section{A. Augmentation de la sécrétion de $K$ par le néphron distal (GTTK} supérieur à 7)

1. Aldostérone

2. Hyperkaliémie

3. Amphotéricine $B$ (?)

4. Anions non réabsorbables (par ex. pénicilline, sulfate, bicarbonate)

\section{B. Augmentation du débit dans le " néphron distal "}

1. Diurétiques (osmotiques ou pharmacologiques)

2. Apport sodique et en eau

3. Glucocorticoïdes dans l'insuffisance surrénalienne (c'est-à-dire l'action de l'aldostérone et/ou le débit tubulaire dans le «néphron distal cortical » [équation l]).

\begin{abstract}
Mesure ponctuelle de la concentration urinaire de $\mathbf{K}$. Un « instantané » de la concentration urinaire de $\mathrm{K}$ est souvent employé comme indice de la réponse rénale à l'hypokaliémie ou à l'hyperkaliémie. Par exemple, on accepte généralement le fait qu'en présence d'une hypokaliémie, une concentration urinaire de $\mathrm{K}$ supérieure à $40 \mathrm{mmol} / \mathrm{l}$ suggère une cause rénale à la perte de $\mathrm{K}$, alors qu'une valeur de moins de $20 \mathrm{mmol} / \mathrm{l}$ oriente vers une cause extrarénale [5]. Cependant, dans ce contexte, les valeurs de la concentration urinaire de $\mathrm{K}$ se retrouvent souvent dans l'intervalle non diagnostique (entre 20 et $40 \mathrm{mmol} / \mathrm{l}$ ). De plus, cette approche ne tient pas compte de la concentration plasmatique de potassium et de la réabsorption médullaire d'eau ni du débit urinaire, qui tous peuvent avoir une grande influence sur les conclusions à tirer de la concentration urinaire de $\mathrm{K}$.
\end{abstract}

Dosages hormonaux. Les dosages de rénine et d'aldostérone sont souvent nécessaires pour établir un diagnostic étiologique précis, mais il s'écoule généralement plusieurs jours avant que les résultats des concentrations hormonales plasmatiques ne soient disponibles.

$\mathrm{m} / \mathrm{s} n^{\circ} 10 \mathrm{v} / \mathrm{ol}$. 4, décembre 88
Un outil diagnostique plus rapide et précis, tenant compte de la réabsorption médullaire d'eau et de la kaliémie, s'avère donc nécessaire dans l'évaluation des désordres de l'homéostasie du potassium. Nous proposons donc ici un nouveau paramètre, fondé sur des principes physiologiques.

\section{Gradient transtubulaire du potassium (GTTK)}

Pour faire une bonne investigation d'un trouble de la kaliémie, il faudrait être à même de mesurer le gradient de potassium établi entre le liquide péritubulaire et la lumière tubulaire du «néphron distal cortical ». Ce gradient, comme il est mentionné précédemment, est le reflet de l'importance de l'activité sécrétoire en K de ce segment. Pour l'apprécier, il nous reste à connaître la concentration luminale en potassium, la concentration péritubulaire étant équivalente à la concentration plasmatique.

En général, le transport net transépithélial du $\mathrm{K}$ est négligeable dans le tubule collecteur médullaire $[6,7]$ de telle sorte que le contenu en $\mathrm{K}$ du liquide tubulaire du « néphron distal cortical » est égal à celui de l'urine. En revanche, la concentration en $\mathrm{K}$ de l'urine variera en fonction de la réabsorption d'eau au niveau médullaire. Comme l'illustre la figure 2, une réabsorption de $75 \%$ du contenu en eau entraîne une concentration urinaire de potassium quatre fois plus élevée que la concentration au niveau du « néphron distal cortical». En présence d'hormone antidiurétique, l'osmolalité du liquide tubulaire au niveau cortical est égale à celle du plasma (mesurée ou égale à $2 \times[\mathrm{Na}]$ + [glucose] + [urée] plasmatiques exprimées en mmol/l) ; connaissant l'osmolalité urinaire, il est facile d'estimer la proportion d'eau réabsorbée par le tubule collecteur médullaire. La concentration urinaire en potassium, divisée par ce facteur de concentration (osmolalité urinaire/osmolalité plasmatique), est donc égale à la concentration luminale en potassium du «néphron distal cortical». Cette dernière valeur divisée par la concentration plasmatique de potassium représente le gradient transtubulaire en potassium (GTTK) :

$$
\begin{aligned}
& \text { GTTK }=[\mathrm{K}]_{\text {urinaire } / \text { osmolalité }} \\
& \text { (urine/plasma)/[K] plasmatiçue (2) }
\end{aligned}
$$

Le GTTK représente une évaluation semi-quantitative de l'activité sécrétoire en $\mathrm{K}$ du « néphron distal cortical ».

La validité de ce calcul de la concentration luminale en potassium du «néphron distal cortical » a été démontrée par microcathétérisme $[6,8]$. Des études similaires ont également établi qu'avec un apport normal de $K$, le transport net transépithélial est faible dans le tubule collecteur médullaire, ainsi que nous en avons fait précédemment l'hypothèse. Toutefois, si l'apport en $\mathrm{K}$ est très faible (hypokaliémie sévère), une faible réabsorption de $\mathrm{K}$ peut entrâेner un changement significatif de sa concentration urinaire[8]. La concentration luminale de $\mathrm{K}$ dans le «néphron distal cortical » sera en conséquence sous-estimée. Malgré cela, le GTTK ne suggèrera pas une cause rénale à l'hypokaliémie si tel n'est pas le cas.

Le GTTK présente donc plusieurs avantages sur les épreuves diagnostiques précédemment décrites. Il est rapidement disponible; il tient compte de la kaliémie et de la réabsorption d'eau au niveau médullaire dans l'interprétation de la concentration urinaire de $\mathrm{K}$; de plus, il permet d'évaluer rapidement l'impact d'un traitement sur la sécrétion de potassium. Ces avantages seront illustrés dans les exemples qui suivent. Il est 
à noter qu'un certain nombre de conditions préalables doivent être respectées pour bien interpréter le GTTK (Tableau II).

\section{Application clinique}

Comme le montre l'équation l, l'excrétion de $\mathrm{K}$ est fonction du volume d'urine et de la concentration urinaire de $K$ (qui dépend surtout de l'activité sécrétoire du « néphron distal cortical » et de la réabsorption d'eau au niveau médullaire). Il est donc utile de pouvoir rapidement obtenir un indice de l'action de l'aldostérone pour évaluer les troubles de l'homéostasie du potassium. Un GTTK supérieur à 7 suggère que le processus sécrétoire de $\mathrm{K}$ est stimulé, le plus souvent par l'effet de l'aldostérone (l'hyperkaliémie peut aussi d'elle-même augmenter la sécrétion de $\mathrm{K}$, tout comme l'amphotéricine $\mathrm{B}$ [8]. En revanche, un GTTK inférieur à 4 suggère que ce processus n'est pas activé de façon significative, ce qui serait inapproprié en présence d'hyperkaliémie et pourrait être secondaire à un hypoaldostéronisme (insuffisance surrénalienne, déficience en rénine ou inhibition de l'enzyme de conversion de l'angiotensine) ou à une diminution de la réponse rénale à l'action de l'aldostérone.

L'interprétation du GTTK, conjointement avec les valeurs simultanément mesurées de rénine et d'aldo- stérone plasmatiques, permet de situer de façon plus adéquate le niveau de dysfonctionnement. Par exemple, si le GTTK est, de façon inadéquate, bas en présence d'hyperkaliémie, on peut soupçonner un défaut de la sécrétion d'aldostérone. On peut alors administrer une dose physiologique de minéralocorticoïde (0,05 mg de $9 \alpha$-fludrocortisone per os) et recalculer le GTTK ; une augmentation du GTTK égale ou supérieure à 7 , deux heures plus tard, suggère une réponse du rein à l'aldostérone. Ceci suggère donc que le GTTK était bas à cause d'une anomalie dans l'axe rénine-aldostérone. En revanche, si le GTTK demeure bas, ceci indique plutôt un trouble de la réponse rénale à l'aldostérone. Par ailleurs, en présence d'hypokaliémie d'origine essentiellement extrarénale, la valeur attendue du GTTK est inférieure à 2 [11]

Exemples hypothétiques. En présence d'une hypokaliémie d'origine extrarénale, le GTTK approprié est inférieur à 4. Toutefois, comme l'illustre la figure 2, cela peut être associé à une concentration urinaire de $\mathrm{K}$ de $40 \mathrm{mmol} / \mathrm{l}$ si la réabsorption médullaire d'eau est élevée. A l'inverse, une concentration urinaire de $\mathrm{K}$ de $20 \mathrm{mmol} / \mathrm{l}$, chez un patient hypokaliémique avec une urine isotonique, peut être considérée, à tort, basse et empêcher de déceler la cause rénale sous-jacente (figure 3). Dans ce

7. Honrath U, Sonnenberg H, Wilson DR, Halperin ML. Role of aldosterone and the plasma potassium concentration in $\mathrm{K}$ transport by the medullary collecting duct. (Soumis en 1987.)

8. West ML, Marsden PA Richarson RMA Zettle RM, Halperin ML. New clinical approach to evaluate disorders of potassium excretion. Min Elect Metab 1986; 12 : 234-8.

9. West ML, Sonnenberg H, Veress A, Halperin ML. The relationship between the plasma potassium concentration and renal potassium excretion in the adrenalectomized rat. Clin Sci $1987 ; 72$ : 577-83.

10. Zettle RM, West ML, Josse RG, Richardson RMA, Marsden PA, Halperin ML. Renal potassium handling during states of low aldosterone bio-activity: a method to differentiate renal and non-renal causes. $\mathrm{Am}$ Nephrol 1987; $7: 360-6$.

11. Ethier J, Sonnenberg H, Lemann J, Halperin M. Evaluation of the renal contribution to $\mathrm{K}$ balance during hypokaliemia. Communication présentée à l'assemblée de l'American

Tableau II

CONDITIONS PRÉALABLES A L'UTILISATION DU GTTK COMME INDICE DE LA SÉCRÉTION DE K AU NIVEAU DU «NÉPHRON DISTAL »

\section{Conditions}

1. Apport de $\mathrm{Na}$ au * néphron distal » ne limitant pas la sécrétion de $K$

2. Présence d'ADH pour permettre la sécrétion de $K$ dans le « néphron distal "

3. Petite proportion de $K$ et d'osmoles* $^{*}$ réabsorbées dans le tube collecteur médullaire

* Note. L'osmolalité urinaire peut refléter moins fidèlement la réabsorption d'eau quand une proportion significative de particules luminales $(\mathrm{Na}, \mathrm{K}, \mathrm{Cl}$ et urée) est réabsorbée durant le passage dans le tube collecteur médullaire, lorsque le débit urinaire est lent. Ceci amènerait à sous-estimer la réabsorption d'eau en utilisant l'osmolalité urinaire, et donc à surestimer le GTTK.
[Na] urinaire supérieure à $25 \mathrm{mmol} / \mathrm{I}$

Osmolalité urinaire non inférieure à celle du plasma

Débit urinaire d'au moins

$0,5 \mathrm{ml} / \mathrm{min}$ Society of Nephrology. Décembre 1988. 
cas, l'absence de réabsorption d'eau par le tubule collecteur médullaire (urine iso-osmolaire) signifie qu'il n'y a pas eu d'augmentation de la concentration de $\mathrm{K}$ durant le passage à travers la médullaire. Notons que la concentration plasmatique de $\mathrm{K}$ doit être incluse dans l'estimation du GTTK. En effet, si celle-ci est de $2 \mathrm{mmol} / \mathrm{l}$, la concentration «tubulaire » maximale de $\mathrm{K}$ sera seulement de $20 \mathrm{mmol} / \mathrm{l}$, avec un GTTK maximal de 10.

De la même façon, si la concentration plasmatique de $\mathrm{K}$ est de $5,5 \mathrm{mmol} / \mathrm{l}$ et le GTTK faible (égal à 4), la concentration luminale de $\mathrm{K}$ dans le «néphron distal cortical » sera de $22 \mathrm{mmol} / \mathrm{l}$. Si la moitié du volume est réabsorbée dans la médullaire (osmolalité urinaire de $600 \mathrm{mosm} / \mathrm{kg} \mathrm{H} \mathrm{H}_{2} \mathrm{O}$ ), la concentration de $\mathrm{K}$ urinaire sera de $44 \mathrm{mmol} / \mathrm{l}$. En ne tenant pas compte de l'osmolalité urinaire, on peut se méprendre en croyant la réponse rénale adéquate. Au contraire, un GTTK égal à 4 en présence d'une hyperkaliémie suggère que l'action rénale de l'aldostérone est insuffisante. A ce stade, l'administration d'une dose physiologique de minéralocorticoïde serait indiquée afin d'évaluer la réponse rénale à cette hormone.

Exemples cliniques. - Une femme de 29 ans, avec volume circulatoire diminué et une hyperpigmentation, est admise dans le service. Sa kaliémie est de $6,8 \mathrm{mmol} / \mathrm{l}$ et sa concentration urinaire de $\mathrm{K}$ de $58 \mathrm{mmol} / \mathrm{l}$ (le Tableau III apporte des données supplémentaires). L'impression clinique d'une insuffisance surrénalienne est-elle appuyée par les données de laboratoire?

A première vue, son GTTK initial, égal à 5 , peut sembler élevé en présence d'une déficience en aldostérone. Toutefois, on sait que l'hyperkaliémie stimule la sécrétion de $\mathrm{K}$ du néphron distal [9] (Tableau I). Après réhydratation par du soluté salé, et administration d'une dose physiologique de remplacement de glucocorticoïde, la kaliémie descend à $5,7 \mathrm{mmol} / \mathrm{l}$ et le GTTK à 2,6 (valeur basse en présence d'hyperkaliémie). Après avoir reçu une dose physiologique de minéralocorticoïde, le GTTK augmente rapidement (supé$\mathrm{m} / \mathrm{s} n^{\circ} 10$ vol. 4 , décembre 88

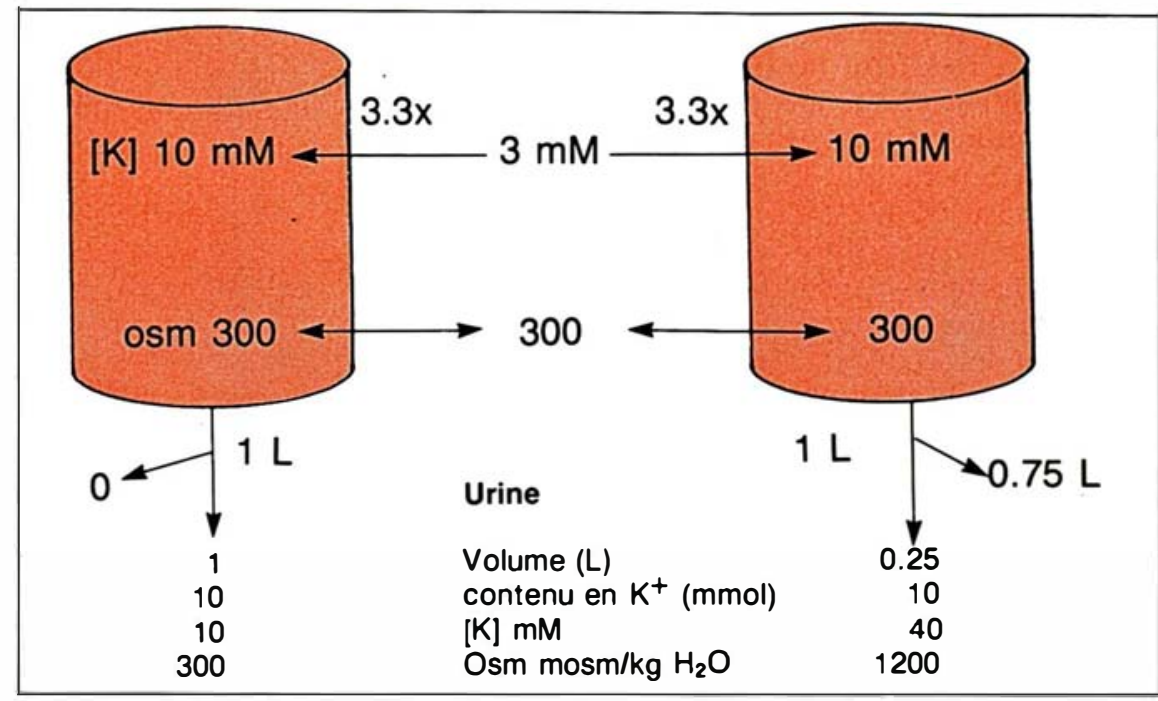

Figure 2. Effet de la réabsorption médullaire d'eau sur la concentration urinaire de $K$. Les structures cylindriques (en rouge) représentent le tubule collecteur cortical et les flèches qui se trouvent au-dessous, le tubule collecteur médullaire. Dans les deux exemples, le gradient transtubulaire de $K$ est faible, c'està-dire que la concentration luminale de $K$ est de $10 \mathrm{mmol} / \mathrm{l}$ ou $3,3 \mathrm{fois} \mathrm{la}$ concentration de $K$ péritubulaire de $3 \mathrm{mmol} / \mathrm{I}$. Pour simplifier, prenons le cas d'un litre de liquide traversant le tubule collecteur médullaire. Dans l'exemple de gauche, il n'y a pas de réabsorption d'eau, alors que $75 \%$ de l'eau est réabsorbée dans l'exemple de droite. Dans les deux cas, il n'y a ni réabsorption ni sécrétion de $K$ dans le tubule collecteur médullaire. Donc, bien que l'excrétion de $K$ soit égale dans les deux cas, la concentration urinaire de $K$ est 4 fois plus élevée là $40 \mathrm{mmol} / \mathrm{I})$ lorsqu'il y a réabsorption d'eau dans la médulla (à droite), ce dont on doit tenir compte dans l'évaluation de la concentration de $K$ urinaire.

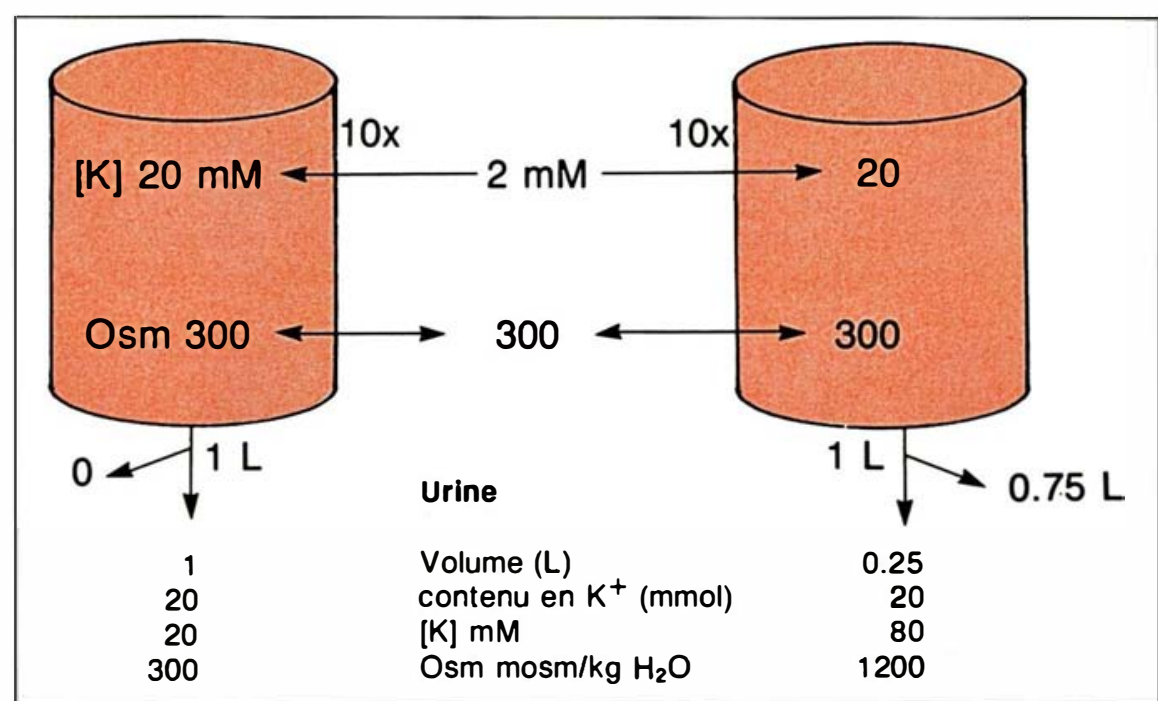

Figure 3. Importance de la concentration plasmatique de $K$ dans l'excrétion de $K$. Voir figure 2 pour les détails. Si l'aldostérone a augmenté le GTTK à 10, la concentration luminale de $K$ peut n'être que de $20 \mathrm{mmol} / \mathrm{l}$ en présence d'hypokaliémie sévère $(2 \mathrm{mmol} / \mathrm{ll})$. S'il n'y a pas de réabsorption d'eau dans le tubule collecteur médullaire, la concentration urinaire de $K$ sera aussi de $20 \mathrm{mmol} / \mathrm{l}$. On doit donc tenir compte de la concentration plasmatique de $K$ dans l'interprétation de la concentration urinaire de $K$. 
rieur à 7) en deux heures, alors que la kaliémie redevient normale, indiquant une réponse rénale normale à cette hormone. Ces résultats confirment clairement que le trouble de l'homéostasie du K est d'origine surrénalienne et illustrent le fait que d'autres facteurs que l'aldostérone peuvent influencer la sécrétion de $\mathrm{K}$ par le « néphron distal cortical ». Ces facteurs sont présentés d'une façon plus détaillée par Field [4], Narins [5] et West $[8,9]$.

- Un alcoolique de 34 ans est admis dans le service pour des vomissements ayant duré plusieurs jours. il présente un déficit important du volume extracellulaire. Les examens de laboratoire révèlent une alcalose métabolique sévère ( $\mathrm{pH}$ sanguin : 7,60 et bicarbonates sériques : $62 \mathrm{mmol} / \mathrm{l}$ ) et u n e h y p o kali é mi e sévè re $(1,9 \mathrm{mmol} / \mathrm{l})([\mathrm{Na}]$ plasmatique de $132 \mathrm{mmol} / \mathrm{l}$ et [Cl] de $57 \mathrm{mmol} / \mathrm{l}$ ). Les valeurs d'électrolytes urinaires à l'admission sont: [Na] : $11 \mathrm{mmol} / \mathrm{l}$; [K] : $12 \mathrm{mmol} / \mathrm{l}$; [Cl] : $27 \mathrm{mmol} / \mathrm{l}$; et une osmolalité de $600 \mathrm{mosm} / \mathrm{kg}$ $\mathrm{H}_{2} \mathrm{O}$. Son GTTK initial est donc de $3,2\left(12 \mathrm{~m} \mathrm{~mol} / \mathrm{l} /\left[\begin{array}{lll}6 & 0 & 0\end{array} / 300\right] /\right.$ $1,9 \mathrm{mmol} / \mathrm{l})$. L'aldostérone, libérée en réponse à la contraction volémique, n'est pas parvenue à élever le GTTK en raison de l'apport réduit de $\mathrm{Na}$ au niveau du «néphron distal cortical » : étant donné la réabsorption médullaire d'eau, la concentration urinaire de $\mathrm{Na}$, égale à $12 \mathrm{mmol} / \mathrm{l}$, représente une concentration de $\mathrm{Na}$ de $6 \mathrm{mmol} / \mathrm{l}$ au niveau du « néphron distal cortical » (voir Tableau II).

Sur le plan thérapeutique, on peut anticiper une perte urinaire en $\mathrm{K}$ importante avec la diurèse alcaline induite par l'expansion volémique (puisque l'aldostérone continuera d'agir pour un certain temps). Dans ce contexte, on a pensé administrer une dose de $10 \mathrm{mg}$ d'amiloride (diurétique bloquant les canaux luminaux de $\mathrm{Na}$ au niveau du «néphron distal cortical » et ainsi la sécrétion de $\mathrm{K}$, figure 1 ) pour minimiser cette perte prévue de $\mathrm{K}$.

En conclusion, l'excrétion rénale de $\mathrm{K}$ est sous la dépendance du débit urinaire et de la concentration urinaire de $\mathrm{K}$. Cette dernière dépend du gradient de concentration de $\mathrm{K}$ engendré à travers l'épithélium du « néphron distal cortical » (GTTK),

\begin{tabular}{|c|c|c|c|}
\hline \multicolumn{4}{|c|}{$\begin{array}{c}\text { Tableau III } \\
\text { DONNÉES DE LABORATOIRE DE L'EXEMPLE CLINIQUE I }\end{array}$} \\
\hline & Admission & $\begin{array}{l}\text { Administration de } \\
\text { glucocorticoïdes }\end{array}$ & $\begin{array}{l}\text { Administration de } \\
\text { minéralocorticoïdes }\end{array}$ \\
\hline \multicolumn{4}{|l|}{ Plasma } \\
\hline $\begin{array}{l}\mathrm{Na}(\mathrm{mmol} / \mathrm{l}) \\
\mathrm{K}(\mathrm{mmol} / \mathrm{I}) \\
\mathrm{Cl}(\mathrm{mmol} / \mathrm{I}) \\
\mathrm{HCO}_{3}^{-}(\mathrm{mmol} / \mathrm{I}) \\
\text { Créatinine }(\mu \mathrm{mol} / \mathrm{I})\end{array}$ & $\begin{array}{c}128 \\
6,8 \\
97 \\
20 \\
140\end{array}$ & $\begin{array}{c}133 \\
5,7 \\
106 \\
19 \\
130\end{array}$ & $\begin{array}{c}133 \\
4,9 \\
104 \\
18 \\
80\end{array}$ \\
\hline \multicolumn{4}{|l|}{ Urine } \\
\hline $\begin{array}{l}\mathrm{Na}(\mathrm{mmol} / \mathrm{l}) \\
\mathrm{K}(\mathrm{mmol} / \mathrm{I}) \\
\mathrm{Osm}\left(\mathrm{mosm} / \mathrm{kg} \mathrm{H}_{2} \mathrm{O}\right)\end{array}$ & $\begin{array}{r}104 \\
58 \\
476\end{array}$ & $\begin{array}{r}81 \\
19 \\
362\end{array}$ & $\begin{array}{r}21 \\
71 \\
463\end{array}$ \\
\hline GTTK & 5,0 & 2,6 & 8,9 \\
\hline
\end{tabular}

stérone. On peut estimer la concentration luminale de $\mathrm{K}$ du «néphron distal cortical » en corrigeant la concentration urinaire de $\mathrm{K}$ pour la réabsorption médullaire d'eau, reflétée par l'augmentation de l'osmolalité urinaire (ceci n'est valable qu'au cours de l'émission d'une urine isoosmolaire ou hyperosmolaire). Par conséquent, le gradient transtubulaire de $\mathrm{K}$ peut être calculé selon l'équation :

$$
\begin{aligned}
& \text { GTYK }=[\mathrm{K}]_{\text {urinaire/osmolalité }} \\
& \text { (urine/plasma)/[K] }]_{\text {plasmatique (2) }}
\end{aligned}
$$

Un GTTK égal ou supérieur à 7 suggère une sécrétion de K stimulée, alors qu'une valeur inférieure à 4 suggère une activité sécrétoire faible. Si le GTTK n'est pas approprié en présence d'une hyperkaliémie, on peut administrer une dose physiologique de minéralocorticoïde pour évaluer la capacité du rein à répondre à cette hormone. Le GTTK est donc un indice utile, facile à obtenir et rapidement disponible, qui permet d'évaluer la réponse rénale aux anomalies de la kaliémie. Son analyse, après administration de minéralocorticoïdes ou conjointement avec les concentrations hormonales (lorsqu'elles sont disponibles), permet de cerner la physiopathologie de ces anomalies

\section{Summary}

To treat patients with disorders of potassium $(\mathrm{K})$ homeostasis, the clinician should determine their etiology. The tests currently used to evaluate renal causes for hypokaliemia or hyperkaliemia often lead to inconclusive or erroneous results. Accordingly, we developed a new test on «spot » urines to help assess whether the K secretory process in the cortical «distal nephron » is activated (partially the result of aldosterone activity). This test is simple, non invasive and is based on physiological principles. It requires the measurement of the $[\mathrm{K}]$ in the plasma and urine as well as the urine osmolality; the latter is required to correct for medullary water abstraction. The transtubular [K] gradient in the cortical «distal nephron » (TTKG) is calculated as follows: TTKG $=[\mathrm{K}]_{\text {urine }}$ / (urine/plasma) osmolality $/[\mathrm{K}]_{\text {plasmar }}$ Values 7 or greater suggest that $K$ secretion is stimulated whereas values less than 4 suggest that basal activity is low. Examples are presented to illustrate the advantages of this approach. 